\title{
Fate of antibiotic resistance genes in reclaimed water reuse system with integrated membrane process
}

\author{
Jian $\mathrm{Lu}^{\mathrm{a}, \mathrm{b}, \mathrm{c}, *}$, Yuxuan Zhang ${ }^{\mathrm{a}, \mathrm{b}}$, Jun $\mathrm{Wu}^{\mathrm{d}}$, Jianhua Wang ${ }^{\mathrm{a}}$, Ying Cai ${ }^{\mathrm{a}}$ \\ ${ }^{a}$ CAS Key Laboratory of Coastal Environmental Processes and Ecological Remediation, Yantai Institute of Coastal Zone Research (YIC), Chinese Academy of Sciences \\ (CAS), Shandong Key Laboratory of Coastal Environmental Processes, YICCAS, Yantai, Shandong 264003, PR China \\ b University of Chinese Academy of Sciences, Beijing 100049, PR China \\ ${ }^{\mathrm{c}}$ Center for Ocean Mega-Science, Chinese Academy of Sciences, 7 Nanhai Road, Qingdao, 266071, PR China \\ ${ }^{\mathrm{d}}$ School of Resources and Environmental Engineering, Ludong University, Yantai, Shandong 264025, PR China
}

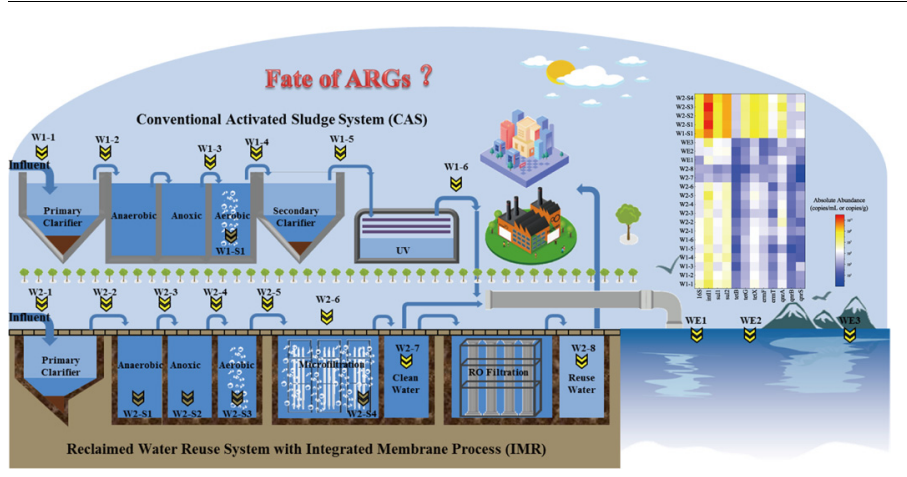

\section{A R T I C L E I N F O}

Editor: Daniel C.W. Tsang

Keywords:

Antibiotic resistance genes

Integrated membrane process

Reclaimed water reuse system

Removal efficiency

Bacterial community

\begin{abstract}
A B S T R A C T
The fate of antibiotic resistance genes (ARGs) in reclaimed water reuse system with integrated membrane process (IMR) was firstly investigated. Results indicated that ARGs, class 1 integrons (intI1) and 16S rRNA gene could be reduced efficiently in the IMR system. The absolute abundance of all detected ARGs in the reuse water after reverse osmosis (RO) filtration of the IMR system was $4.03 \times 10^{4}$ copies $/ \mathrm{mL}$, which was about 2-3 orders of magnitude lower than that in the raw influent of the wastewater treatment plants (WWTPs). Maximum removal efficiency of the detected genes was up to $3.8 \mathrm{log}$ removal values. Daily flux of the summation of all selected ARGs in the IMR system decreased sharply to $(1.02 \pm 1.37) \times 10^{14}$ copies/day, which was $1-3$ orders of magnitude lower than that in the activated sludge system (CAS) system. The strong clustering based on ordination analysis separated the reuse water from other water samples in the WWTPs. Network analysis revealed the existence of potential multi-antibiotic resistant bacteria. The potential multi-antibiotic resistant bacteria, including Clostridium and Defluviicoccus, could be removed effectively by microfiltration and RO filtration. These findings suggested that the IMR system was efficient to remove ARGs and potential multi-antibiotic resistant bacteria in the wastewater reclamation system.
\end{abstract}

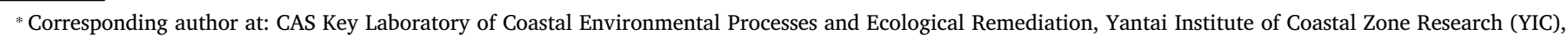
Chinese Academy of Sciences (CAS), Shandong Key Laboratory of Coastal Environmental Processes, YICCAS, Yantai, Shandong 264003, PR China.

E-mail addresses: jlu@yic.ac.cn, lujianleo@163.com (J. Lu). 


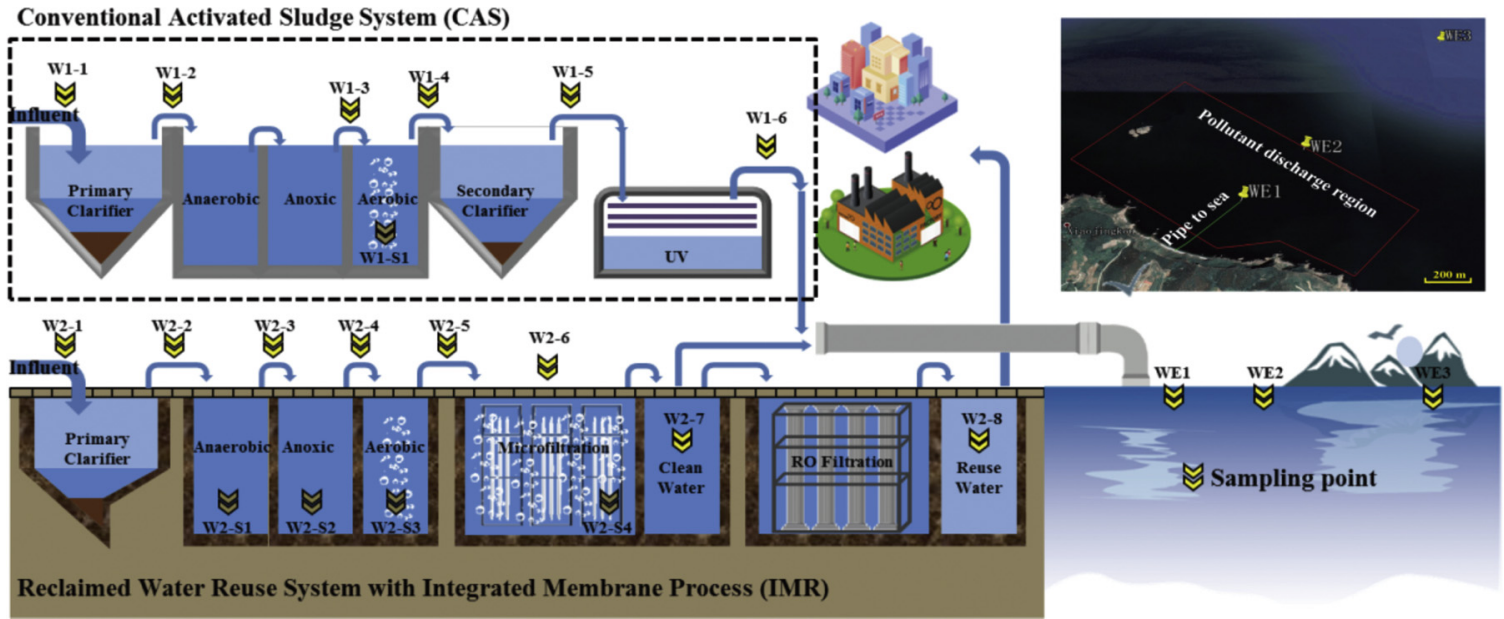

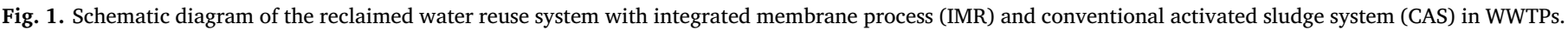
Upper-right corner, the map of seawater sampling locations.

\section{Introduction}

Extensive and improper use of antibiotics in hospital, livestock farming and agriculture-industrial production has led to the rapid prevalence of antibiotic resistant bacteria (ARB) and antibiotic resistance genes (ARGs), even the widespread occurrence of multi-antibiotic resistant bacteria (MARB) and genes in various environments (Zhu et al., 2017; Zhou et al., 2011; Zhang et al., 2015; Su et al., 2017). ARGs might persist and disseminate widely by horizontal transfer mechanism in the environment even in the absence of environmental selection pressure. Numerous studies have reported the occurrence, distribution and removal of ARGs in different environments ( $\mathrm{Lu}$ et al., 2019). The increasing emergence and propagation of antimicrobial resistance have been posing risks to ecological sustainability and human health (Berendonk et al., 2015).

Many developing countries are facing the challenge of water shortages. Reclaimed water has been identified as a feasible and effective solution to water shortages (Wang et al., 2014). However, the lack of water quality assurance remains one of the most serious hurdles for the wide use of reclaimed water. As emerging contaminants, ARGs have become one of the important factors effecting water quality (Wang et al., 2014; Hu et al., 2018). Reclaimed water derived from wastewater treatment plants (WWTPs) can relieve the serious challenge to some extent. WWTPs, the important receiver of domestic wastewater, medical wastewater, pharmaceutical wastewater and husbandry wastewater, have played critical roles in removing ARGs to alter the distribution and abundance of ARGs in receiving environment (Tahrani et al., 2015; Proia et al., 2018). WWTPs not only serve as important reservoirs of persistent ARGs and ARB from different sources, but also play vital roles in reducing and controlling the proliferation and spread of ARB and ARGs (Pruden et al., 2013). However, the conventional activated sludge (CAS) system in WWTPs cannot completely remove ARGs and ARB, and it was influenced by different treatment processes. Therefore, the advanced treatment after conventional biological process and disinfection should be applied for improving the removal efficiency of emerging contaminants (Michael et al., 2013), including reverse osmosis membrane filtration, activated oxidation processes, activated carbon adsorption, chlorination, ultraviolet and ozone disinfection, etc (Zheng et al., 2017). Many techniques such as coagulation (Li et al., 2017), anaerobic treatment (Yi et al., 2017; Ma et al., 2018), ultraviolet disinfection (Hu et al., 2016; Zhang et al., 2019), advanced chemical oxidation (Michael-Kordatou et al., 2018; Fiorentino et al., 2019), and membrane bioreactor system (Le et al., 2018) have been used on the elimination of ARGs. Zhang et al. (2016) discovered that Fenton oxidation process $\left(\mathrm{Fe}^{2+} / \mathrm{H}_{2} \mathrm{O}_{2}\right)$ was effective on the reduction of ARGs.
However, cost of this technique was high due to consumption of large amounts of reagents and it also brought secondary pollutants. Several studies found that the removal of ARGs in membrane bioreactor (MBR) was better than that in traditional processes (Le et al., 2018). However, systematic analysis and comparisons for the removal of ARGs by these methods have not been reported.

Membrane treatment is one of the promising solutions for removal of emerging contaminants. Theoretically, ARGs as high molecular compounds could be trapped and removed by advanced membrane. Lan et al. (2019) found that nanofiltration and reverse osmosis filtration processes were capable of removing various types of ARGs efficiently. However, limited studies are available on investigating the removal of ARGs and ARB in reclaimed water reuse system with integrated membrane process (IMR) consisting of conventional anaerobic-anoxicaerobic (AAO) process, microfiltration, ultrafiltration, and reverse osmosis treatment. Therefore, the objective of this study was to evaluate the distribution and removal efficiency of ARGs and class 1 integron (intI1) in IMR system, microfiltration membrane bioreactor (MBR) system and conventional activated sludge system (CAS) in WWTPs. Previous studies demonstrated that antibiotics and corresponding genes encoding resistance to sulfonamides, tetracyclines, macrolides and quinolones have been detected frequently in the coastal zone of China. Therefore, tetracycline resistance genes (tet $\mathrm{B}$, tet $\mathrm{G}$ and tet $\mathrm{X}$ ), sulfonamide resistance genes (sul1 and sul2), macrolide resistance genes (ermF and $e r m \mathrm{~T}$ ), quinolone resistance genes ( $q n r \mathrm{~A}, q n r \mathrm{~B}$ and $q n r S$ ), intI 1 and 16S rRNA gene were investigated and quantified by real-time PCR.

\section{Materials and methods}

\subsection{Sample collection}

A typical WWTPs combining the CAS system with the IMR system in Yantai City, was chosen to investigate the removal of ARGs. Besides, the effluent of the WWTPs and seawater collected from the receiving sea were monitored for investigating the influence of the WWTPs on receiving environment. Six wastewater samples (W1-1, W1-2, W1-3, W14, W1-5 and W1-6) and one sludge sample (W1-S1) were collected from the CAS system, eight water samples (W2-1, W2-2, W2-3, W2-4, W2-5, W2-6, W2-7 and W2-8) and four sludge samples (W2-S1, W2-S2, W2-S3 and W2-S4) were collected from the IMR system, and three seawater samples (WE1, WE2 and WE3) were collected from the receiving sea (Fig. 1). Samples were collected in sextuplicate from each sampling point, and two of them were mixed well as one subsample. Subsample1 , subsample- 2 and subsample- 3 were parallel samples in each sampling site. Subsequent DNA extraction, PCR amplification and statistical 
analysis were performed base on the three parallel samples, while the high-throughput sequencing was conducted based on the mixture of subsample-1, subsample-2 and subsample-3. WE1, WE2 and WE3 were collected from the receiving sea. WE1 was located at the drainage outlet of the WWTPs, WE2 was at the edge of pollutant discharge region of the WWTPs, and WE3 was about $2.0 \mathrm{~km}$ away from the outlet of WWTPs. Sludge samples were collected from aerobic tank (W1-S1) of the CAS system, anaerobic tank (W2-S1), anoxic tank (W2-S2), aerobic tank (W2-S3), and microfiltration tank (W2-S4) of the IMR system. All water samples and sludge samples were stored in sterile amber containers with iceboxes and transported immediately to laboratory for further analyses within the same day.

\subsection{Physicochemical analysis}

Salinity was measured using a portable refractometer (LH-Y100, Lohand Biological, China) and $\mathrm{pH}$ was determined by a $\mathrm{pH}$ meter (PHS3C, INESA, China). Ammonia, nitrates, nitrites, phosphates, silicate, total nitrogen (TN) and total phosphorus (TP) were analyzed by a continuous flow analyzer (Auto Analyzer III, Seal, Germany). Total organic carbon (TOC) was measured with a total organic carbon analyzer (TOC-VCPH, Shimadu, Japan). Prior to analyses, water samples were filtered through $0.45 \mu \mathrm{m}$ mixed cellulose esters membranes (Merck Millipore Ltd, Ireland). The water quality parameters were shown in Table S1.

\subsection{DNA extraction}

Water sample with the volume of $1.0 \mathrm{~L}$ collected from each sampling point was filtered through $0.22 \mu \mathrm{m}$ mixed cellulose esters membranes (Millipore) for DNA extraction according to previous studies (Lu et al., 2019; Hu et al., 2018), and $0.5 \mathrm{~g}$ sludge from each sample was used for DNA extraction. Extraction of DNA was performed by TIANamp Soil DNA Kit (TIANGEN Biotech, Beijing, China) according to manufacturer's instructions. Concentration and purity of DNA were measured by a NanoDrop UV-vis spectrophotometer (NanoDrop Lite, Thermo Scientific, Wilmington, USA), and the DNA quality was determined by $1 \%$ agarose gel electrophoresis.

\subsection{Quantification of ARGs}

A total of twelve genes were quantified by real-time PCR ( $q P C R$ ), including 16S rRNA gene, intI1 and ten types of ARGs. All target ARGs conferred resistance to common antibiotic families used in veterinary and human medicine including sulfonamide resistance genes (sul1, sul2), tetracycline resistance genes (tet $\mathrm{B}$, tet $\mathrm{G}$, tet $\mathrm{X}$ ), macrolide resistance genes (ermF, erm T) and quinolone resistance genes (qnrA, qnrB, $q n r S)$. These target ARGs were detected with higher detection frequencies and higher abundances in our previous studies. All primer sequences used in this study were summarized in Table S2, which were reported in previous publications (Zhu et al., 2013).

Calibration standard curves for absolute quantification and positive controls were established by a ten-fold dilution series of plasmids DNA ranging from $10^{7}$ to $10^{0}$ copies/ $\mu \mathrm{L}$, which were extracted from $E$. coli DH5 $\alpha$ as reported previously (Luo et al., 2010). All the gene standards were run together with samples in 384-well plates and each reaction was conducted in triplicate. Each $10 \mu \mathrm{L}$ real-time PCR reaction contained 5.0 $\mu \mathrm{L}$ SYBR Premix Ex Taq II (Takara, Dalian, China), $0.4 \mu \mathrm{L}$ forward primers and $0.4 \mu \mathrm{L}$ reverse primers $(0.4 \mu \mathrm{M})$, and $1.0 \mu \mathrm{L}$ DNA template. Real-time PCR amplified reactions were conducted using BioRad qPCR system (Bio-Rad CFX384 Touch, CA, USA) with the following program: $30 \mathrm{~s}$ for denaturation at $95^{\circ} \mathrm{C}, 40$ cycles of $5 \mathrm{~s}$ at $95^{\circ} \mathrm{C}, 30 \mathrm{~s}$ at annealing temperature and $40 \mathrm{~s}$ for extension at $72^{\circ} \mathrm{C}$. Finally, a melting curve was conducted with temperature ranging from $60^{\circ} \mathrm{C}$ to $95^{\circ} \mathrm{C}$ and the melting curves with a single peak were considered as specific amplification. Reactions with amplification efficiencies ranged from $90 \%$ to $110 \%$ and the $\mathrm{R}^{2}$ values of standard curves more than 0.99 were taken into account for ARGs quantification.

\subsection{Removal efficiencies of $16 S$ rRNA gene, intI1 and ARGs}

Removal efficiencies of 16S rRNA gene, intI1 and ARGs for the CAS system and the IMR system were calculated in equation as follows:

Removal efficiency of CAS system $=\lg \frac{\mathrm{c}_{\mathrm{w} 1-1}}{\mathrm{c}_{\mathrm{w} 1-6}}$

Removal efficiency of MF system $=\lg \frac{\mathrm{c}_{\mathrm{w} 2-1}}{\mathrm{c}_{\mathrm{w} 2-7}}$

Removal efficiency of IMR system $=\lg \frac{c_{\mathrm{w} 2-1}}{c_{\mathrm{w} 2-8}}$

The removal efficiencies of detected genes were assessed using $\log 10$ removal value (LRV) as depicted in Eqs. (1)-(3). The removal efficiency of the CAS system was calculated by Eq. (1). Eq. (2) demonstrated the removal efficiency of the wastewater treatment flow from the raw influent to the effluent of microfiltration in the IMR system. The total removal efficiency of the IMR system was depicted by Eq. (3), ranging from the raw influent to the reuse water.

\subsection{Illumina MiSeq sequencing and analyses of $16 S$ rRNA gene}

Purified microbial DNA extracted from 17 water samples and 5 sludge samples were sent to Majorbio (Shanghai, China) for highthroughput sequencing. The V4-V5 region of the bacterial 16S rRNA gene was selected for amplification with primers 515 F (5'-GTGCCAG CMGCCGCGG-3') and 907R (5'-CCGTCAATTCMTTTRA GTTT - $3^{\prime}$ ) (Wang et al., 2018), using thermocycler PCR system (GeneAmp 9700, ABI, USA). PCR reactions were conducted using the following program: 3 min of denaturation at $95^{\circ} \mathrm{C}, 27$ cycles of $30 \mathrm{~s}$ at $95^{\circ} \mathrm{C}, 30 \mathrm{~s}$ for annealing at $55^{\circ} \mathrm{C}$, and $45 \mathrm{~s}$ for elongation at $72^{\circ} \mathrm{C}$, and a final extension at $72{ }^{\circ} \mathrm{C}$ for $10 \mathrm{~min}$. PCR reactions were performed in triplicate. Each $20 \mu \mathrm{L}$ mixture containing $4 \mu \mathrm{L}$ of $5 \times$ FastPfu Buffer, $2 \mu \mathrm{L}$ of $2.5 \mathrm{mM}$ dNTPs, $0.8 \mu \mathrm{L}$ of each primer $(5 \mu \mathrm{M}), 0.4 \mu \mathrm{L}$ of FastPfu Polymerase and $10 \mathrm{ng}$ of template DNA. The PCR products were extracted from $2 \%$ agarose gels and further purified using the AxyPrep DNA Gel Extraction Kit (Axygen Biosciences, Union City, CA, USA). Purified amplicons were pooled in equimolar and paired-end sequenced $(2 \times 300)$ on an Illumina MiSeq platform (Illumina, San Diego, USA) according to the standard protocols by Majorbio (Shanghai, China). A total of 989,644 high quality $16 \mathrm{~S}$ rRNA gene sequences were generated from the 22 samples. After subsampling to an equal sequence, 2084 operational taxonomic units (OTUs) at $97 \%$ identity were obtained. Raw reads were deposited into the National Center for Biotechnology Information (NCBI) Sequence Read Archive (SRA) database (Accession Number: SRR8490435 - SRR8490453, SRR8504322 - SRR8504324).

\subsection{Statistical analysis}

Statistical analysis and assessments were performed using Origin 2019 (Origin Lab Corporation, USA) and SPSS 19 (IBM, USA). All statistical tests were considered significant at $p<0.05$. Canonical correspondence analysis (CCA) and non-metric multi-dimensional scaling (NMDS) analysis between water quality parameters, antibiotic resistance genes and bacterial communities were performed in $\mathrm{R}$ environment using vegan and ggplot2 packages. Network analysis was performed in R using vegan, igraph and hmisc packages (Li et al., 2015) and further visualized by Cytoscape 3.7.1 to demonstrate the correlation between detected genes and bacterial communities. 


\section{Results and discussion}

\subsection{Fate of ARGs in the effluent of the WWTPs and the receiving environment}

In order to investigate the influence of WWTPs discharge on its receiving environment, the effluent of CAS system (W1-6), the effluent of microfiltration in IMR system (W1-7), and three seawater samples collected from the surrounding sea (WE1, WE2, WE3) were chosen for detection and quantification of target ARGs (sul1, sul2, tet $\mathrm{B}$, tet $\mathrm{G}$, tet $\mathrm{X}$, ermF, erm T, qnrA, qnrB, qnrS), intI1 and 16S rRNA gene. All 12 target genes were detected in these five samples, except that qnrS was detected in the effluent of microfiltration (W2-7) with low abundance of 0.46 copies $/ \mathrm{mL}$. The sul genes (sul1 and sul2), encoding sulfonamide resistant dihydropteroate synthase (Zhang et al., 2014), were the most prevalent ARGs in these water samples with abundances ranging from $2.14 \times 10^{3}$ to $2.25 \times 10^{6}$ copies/mL. The average abundances of tet genes (tet $\mathrm{B}$, tet $\mathrm{G}$ and tet $\mathrm{X}$ ), which encoded tetracycline resistance protein, were lower than those of sul genes but higher than those of $q n r$ and erm genes (Tukey HSD Post-hoc Test, $\mathrm{p}<0.05)$. The qnr genes (qnrA, $q n r \mathrm{~B}$ and $q n r S$ ), encoding resistant to quinolones (Marti et al., 2014), were detected with abundances ranging from 46.0 to $2.48 \times 10^{5}$ copies/mL. The abundances of $q n r \mathrm{~A}$ were higher than those of $q n r \mathrm{~B}$ and $q n r S(\mathrm{p}<0.05)$. The abundances of erm genes (ermF and ermT) were the lowest, which associated with those of methylase conferring macrolides resistance.

As shown in Fig. 2, the absolute copy numbers of sul1, sul2, tetX and qnrA were relatively high, ranging from $10^{3}$ to $10^{8}$ copies/mL. Except erm $\mathrm{T}$ and $q n r \mathrm{~B}$ genes, the absolute abundances of other ARGs in the effluent of microfiltration (W2-7) were significantly lower than those in the effluent of CAS system (W1-6) ( $\mathrm{p}<0.05)$. The absolute abundances of most ARGs (sul, tet, qnrA and qnrS) detected in seawater (WE1, WE2, WE3) were significantly higher than those in the effluent of microfiltration (W2-7), while they were lower than the abundances of genes detected in the effluent of CAS system (W1-6) $(\mathrm{p}<0.05)$

The gene of IntI1 was important transmissible vector that played important role in the emergence and horizontal transfer of ARGs in different environment (Xu et al., 2017). The intI1 gene was prevalent in the water samples in this research. The absolute abundances of intI1 detected in the receiving seawater ranged from $1.48 \times 10^{5}$ to $3.36 \times 10^{7}$ copies $/ \mathrm{mL}$, higher than that in the effluent of microfiltration process $\left(1.13 \times 10^{4}\right.$ copies $\left./ \mathrm{mL}\right)(\mathrm{p}<0.05)$ and comparable with that in the discharge of the CAS system $\left(8.87 \times 10^{6}\right.$ copies $\left./ \mathrm{mL}\right)$ ( $p>0.05$ ). In addition, the abundances of 16S rRNA gene,

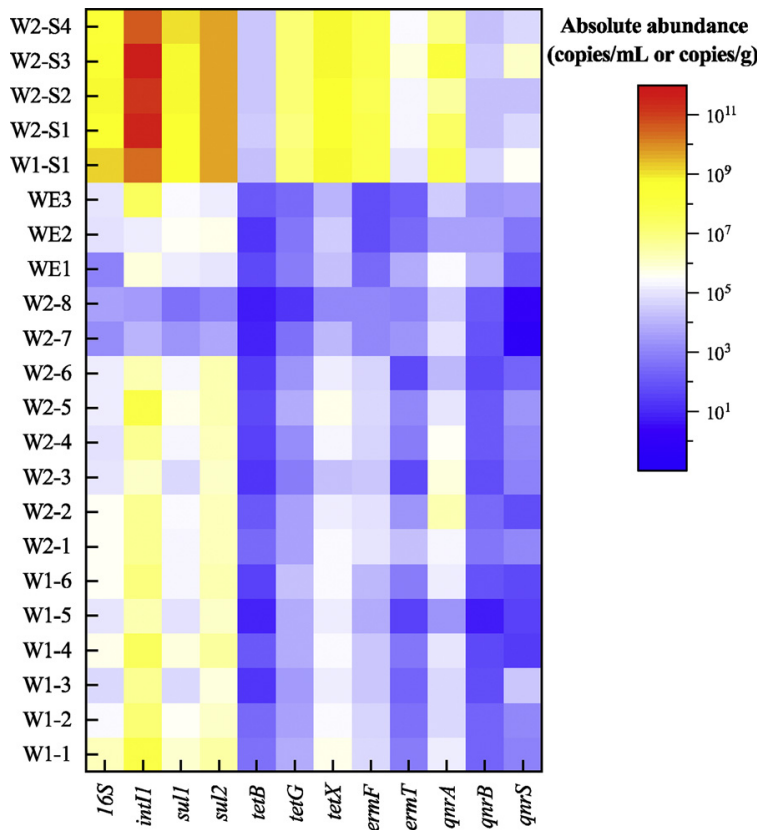

Fig. 3. Heat map of absolute abundances of ARGs, $16 \mathrm{~S}$ rRNA and intI1 in water samples and sludge samples. Copies/mL, unit of water samples; copies/g, unit of sludge samples.

representing the microbial concentrations (Chen and Zhang, 2013), were obviously higher in the effluent of CAS system than those in the effluent of microfiltration system ( $p<0.05$ ). Besides, the abundances of $16 \mathrm{~S}$ rRNA gene in seawater samples ranged from $10^{3}$ to $5.0 \times 10^{6}$ copies/mL, showing lower abundances than those in the effluent of CAS system $(\mathrm{p}<0.05)$.

It has been reported in our previous studies that the abundances of ARGs detected in coastal areas were obviously higher than those in the seawater (Lu et al., 2019). According to the investigation, the abundances of ARGs detected in W1-6 and WE $(1,2,3)$ were 3-6 orders of magnitude higher than that in the seawater, and 1-3 orders of magnitude higher than that in the coastal water. It could be speculated that the area near the drainage outlet of the WWTPs was influenced by the effluent of WWTPs. The discharges of the WWTPs might contribute to the spread of intI 1 and ARGs into surrounding environment. Meanwhile, in comparison with the CAS system, the abundance of ARGs and intI1 decreased obviously after the membrane filtration in the IMR

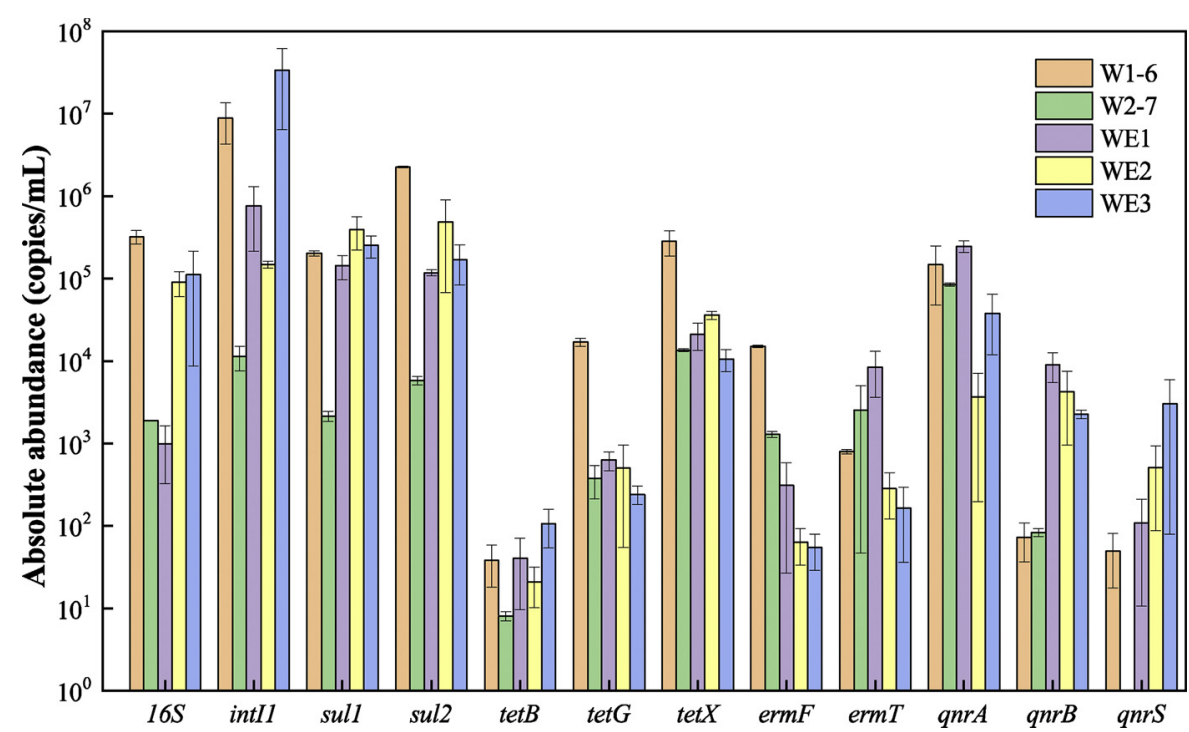

Fig. 2. Absolute abundances (copies/mL) of $16 \mathrm{~S}$ rRNA, intI 1 and ARGs. W1-6, the effluent of conventional activated sludge (CAS) system; W2-7, the effluent after microfiltration in the reclaimed water reuse system with integrated membrane process (IMR); WE1, WE2 and WE3, seawater samples collected from three sampling points located in the receiving sea, approximately $0 \mathrm{~km}, 0.5 \mathrm{~km}$ and $2 \mathrm{~km}$ away from the WWTPs discharge point. 

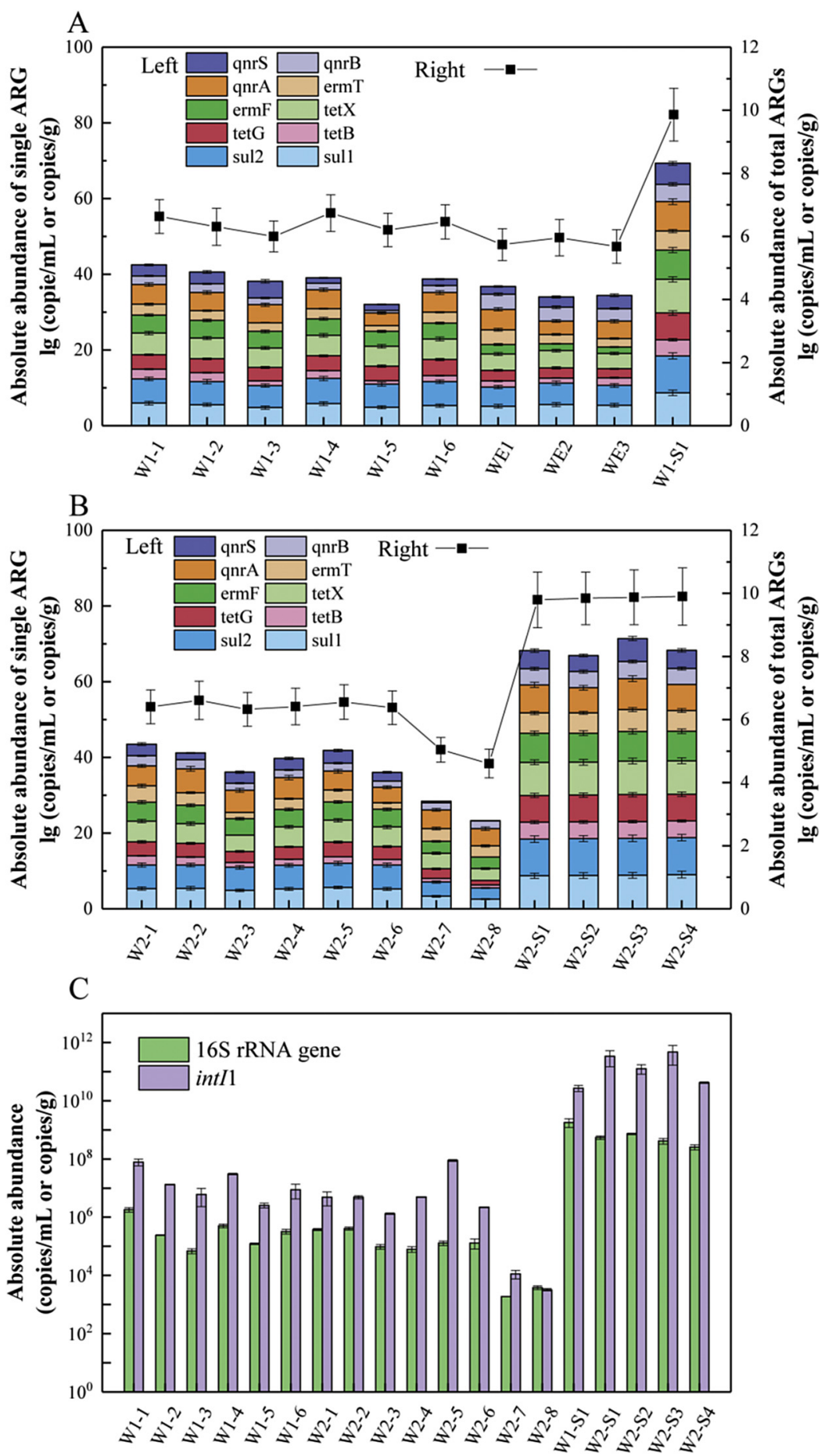

Fig. 4. Log10 value of absolute abundances of ARGs, $16 \mathrm{~S}$ rRNA and intI1 in water samples and sludge samples. A \& B, ARGs in WWTPs and receiving sea (WE1, WE2, WE3). C, 16S rRNA gene and intI1 in WWTPs and WE $(1,2,3)$. Copies/mL, unit of water samples; copies/g, unit of sludge samples.

system.

\subsection{Removal of ARGs and intI1 in the IMR system}

To further investigate the removal of ARGs and intI1 in the IMR system which included anaerobic-anoxic-aerobic (AAO) tank, microfiltration (MF) process and RO filtration process, the absolute abundances of 16S rRNA gene, intI1 and four types of ARGs in this system were quantified. CAS system including AAO tank, secondary clarifier (SC) tank and ultraviolet (UV) disinfection process was used as the comparison. The absolute abundances of detected genes were demonstrated in the heat map (Fig. 3) and bar chart (Fig. 4).

In the raw influent (W1-1 and W2-1) of the WWTPs, all target genes were detected at relatively high abundances. The absolute abundances of ARGs ranged from $3.46 \times 10^{2}$ (tetB) to $2.58 \times 10^{6}$ (sul2) copies $/ \mathrm{mL}$. Sulfonamide resistance genes (sul1 and sul2) were the most prevalent ARGs in the raw influent, followed by tetracycline resistance gene (tetX) and quinolone resistance gene $(q n r A)$. IntI1 gene was detected at the highest abundance up to $7.91 \times 10^{7}$ copies/mL. As shown in Figs. 3 and 4, there was no significant reduction of ARGs detected in the effluent of primary clarify, indicating a low removal efficiency of clarify process ( $p>0.05$ ). AAO tank was indispensable and basic part in sewage 
A
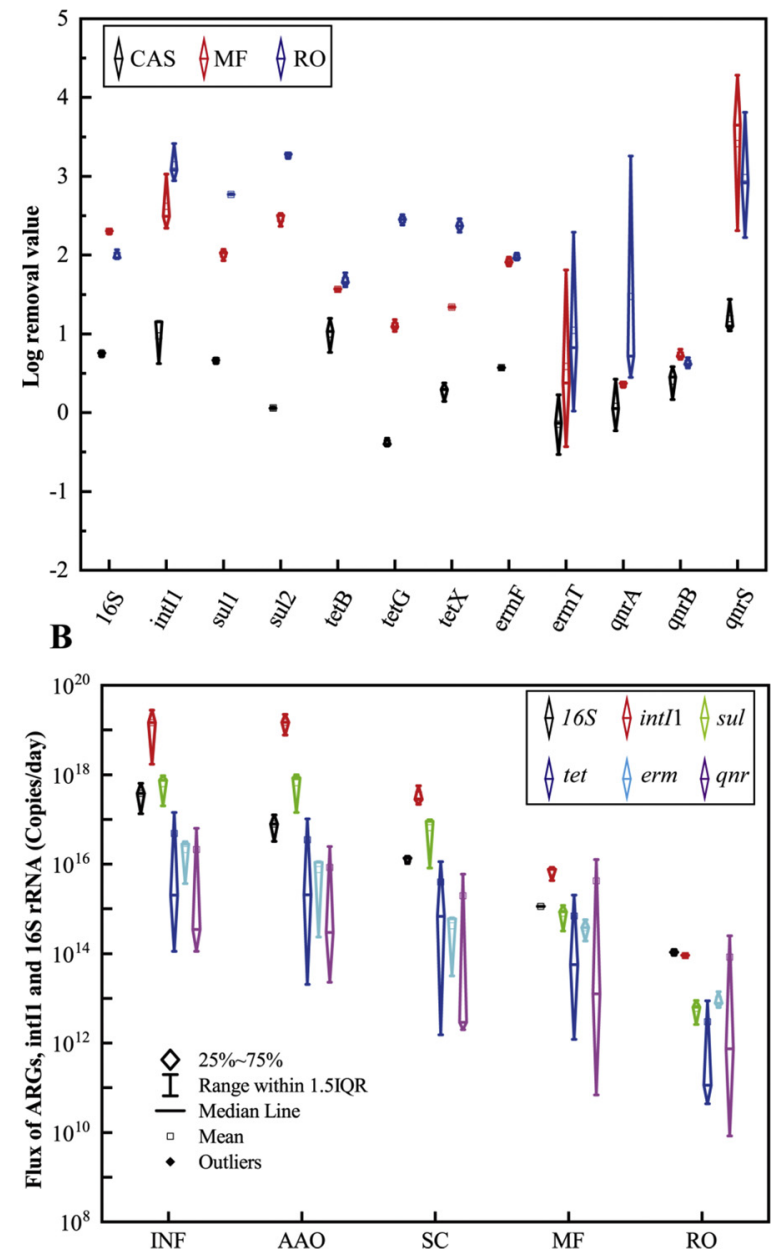

Fig. 5. Removal efficiency (A) and daily flux (B) of ARGs, 16S rRNA and intI1 in the conventional activated sludge system (CAS) and the reclaimed water reuse system with integrated membrane process (IMR). INF, influent of the WWTPs; AAO, anaerobic-anoxic- aerobic tank; SC, secondary clarified tank; MF, microfiltration process; RO, reverse osmosis filtration process.

disposal process to play important role in the removal of a variety of contaminant. It has been reported that enhanced hydrolysis could enable efficient anaerobic treatment of tetracycline production wastewater, with significantly lower generation of ARGs (Yi et al., 2017). However, tetracycline resistance genes could still be detected with abundances of $10^{2}-10^{6}$ copies $/ \mathrm{mL}$ in the water after conventional sewage treatment (Auerbach et al., 2007). In this research, the effluents of AAO process (W1-4 and W2-5) were selected to investigate the removal efficiency of AAO process. The absolute abundances of intI1, 16S rRNA gene and ARGs didn't decrease obviously from the raw influent and the effluent of primary clarify process to the effluent of AAO treatment ( $\mathrm{p}>0.05)$. The result in previous research (Auerbach et al., 2007) was consistent with the result in our research, and provided a good explanation for the high abundances of tet genes $\left(10^{2}-10^{6}\right.$ copies/ $\mathrm{mL}$ ) in the effluent after CAS treatment in our study. Additionally, the abundances of these genes detected in sludge samples were about 3-6 orders of magnitude higher than those detected in corresponding wastewater samples, which demonstrated that sludge was the dominant reservoir for ARGs and microorganism. In the CAS system, the processes of secondary clarify and ultraviolet (UV) disinfection were usually the last two water treatment plants. The abundances of selected ARGs, intI1 and 16S rRNA gene detected in the water after secondary clarify decreased about 1-2 orders of magnitude. However, the abundances of these genes detected in the effluent of UV disinfection increased by 1-2 orders of magnitude on the contrary, which were at the same level with those in the discharge of aerobic tank. In total, there was no significant removal of the detected genes in the CAS system ( $p>0.05$ ).

To investigate the removal efficiencies of ARGs, intI1 and 16S rRNA gene in the IMR system, the effluent after microfiltration (W2-7) and RO filtration (W2-8) was chosen to detect the target genes (Fig. 4). After the microfiltration process, the absolute abundances of sul genes (sul1 and sul2) and qnrS gene decreased with 2-3 orders of magnitude. Abundances of sul1 and sul2 decreased from $1.86 \times 10^{5}$ to $2.14 \times 10^{3}$ copies/mL and from $1.98 \times 10^{6}$ to $5.76 \times 10^{3}$ copies $/ \mathrm{mL}$, respectively, while the abundance of qnrS decreased from 210.33 to 0.46 copies $/ \mathrm{mL}$. Meanwhile, abundances of tet $($ tet $\mathrm{B}, \operatorname{tet} \mathrm{G}$, tet $\mathrm{X})$, erm $(\operatorname{ermF})$ and $q n r$ ( $q n r S$ ) genes decreased with 1-2 orders of magnitude. Moreover, no significant reduction in the abundances of erm $\mathrm{T}$, qnrA and $q n r \mathrm{~B}$ across microfiltration process occurred ( $\mathrm{p}>0.05$ ). RO membrane permeate technology was vital and indispensable unit in the IMR system, which played an important role in desalination and demineralization of water reuse system (Coutinho de Paula and Amaral, 2017). After RO filtration, the absolute abundances of all detected genes (except $q n r \mathrm{~B}$, qnrS and 16S rRNA gene) decreased by one order of magnitude. In the effluent of RO filtration, the abundances of sul, tet, erm, qnr, intI1 and 16S rRNA gene were low by varying from 1.18 to $3.86 \times 10^{3}$ copies $/ \mathrm{mL}$. The total copy number of all selected ARGs was only $4.03 \times 10^{4}$ copies $/ \mathrm{mL}$, which was about 2-3 orders of magnitude lower than that in the raw influent of the WWTPs. Interestingly, the absolute abundances of several ARGs and intI1 in the samples from the influent of the WWTPs (W21) to the effluent after microfiltration (W2-7) were higher than those of 16S rRNA gene. The rational explanation was that the extracellular DNA (eDNA) in water except the bacteria might survive after the common water treatment technologies such as AAO treatment and microfiltration (Mao et al., 2014). However, the absolute abundances of most of ARGs and intI1 in the effluent after RO filtration (W2-8) were lower than those of 16S rRNA gene, suggesting that the eDNA might be removed efficiently through the RO filtration.

Removal efficiencies were calculated by log removal value of the raw influent and the effluent after water treatment. As shown in Fig. 5A, the removal efficiencies of sul (sul1 and sul2) genes were up to 2.1 and $2.5 \log$ removal values after microfiltration process, and up to 2.8 and $3.3 \log$ removal values after RO filtration in the IMR system. However, the log removal values of sul1 and sul2 genes were only about $0.65 \pm 0.04$ and $0.06 \pm 0.01$ in the CAS system, respectively. For tet genes (tet $\mathrm{B}$, tet $\mathrm{G}$, tet $\mathrm{X}$ ), the $\log$ removal values ranged from 1.6 to 2.5 after RO filtration and ranged from 1.0 to 1.6 after microfiltration. In addition, the removal efficiencies of erm (ermF, ermT) and qnr (qnrA, $q n r \mathrm{~B}, q n r \mathrm{~S})$ genes were up to 2.3 and $3.8 \log$ removal values after the permeate of RO membrane, and up to 2.0 and 4.3 log removal values after the permeate of microfiltration membrane. As control, the removal efficiencies of erm and qnr genes were poor in the CAS system, lower than $1.4 \log$ removal values. In summary, the removal efficiencies of detected genes after RO filtration were the highest, followed by the removal efficiencies after microfiltration in IMR system. The removal efficiencies of selected genes after CAS treatment were the lowest.

Daily flux of ARGs, intI 1 and 16S rRNA gene through the IMR system and the CAS system were illustrated in Fig. 5B. Results indicated that the raw influent of the WWTPs was severe breeding ground for ARGs, intI1 and 16S rRNA gene by holding the highest daily flux of each detected gene. Total daily flux of all detected ARGs was up to $(7.22 \pm 4.16) \times 10^{17}$ copies/day. Daily flux in the effluent of AAO process was the secondary (sum to $(7.16 \pm 4.83) \times 10^{17}$ copies/day) to the raw influent, followed by the unit of secondary clarify (SC, $(7.19 \pm 5.05) \times 10^{17}$ copies/day). Compared with the CAS system, daily flux of the summation of all detected ARGs decreased to $(6.09 \pm 0.63) \times 10^{15}$ copies/day in microfiltration unit and decreased sharply to $(1.02 \pm 1.37) \times 10^{14}$ copies/day in RO filtration process of the IMR system, which were 1-3 orders of magnitude lower than that in 

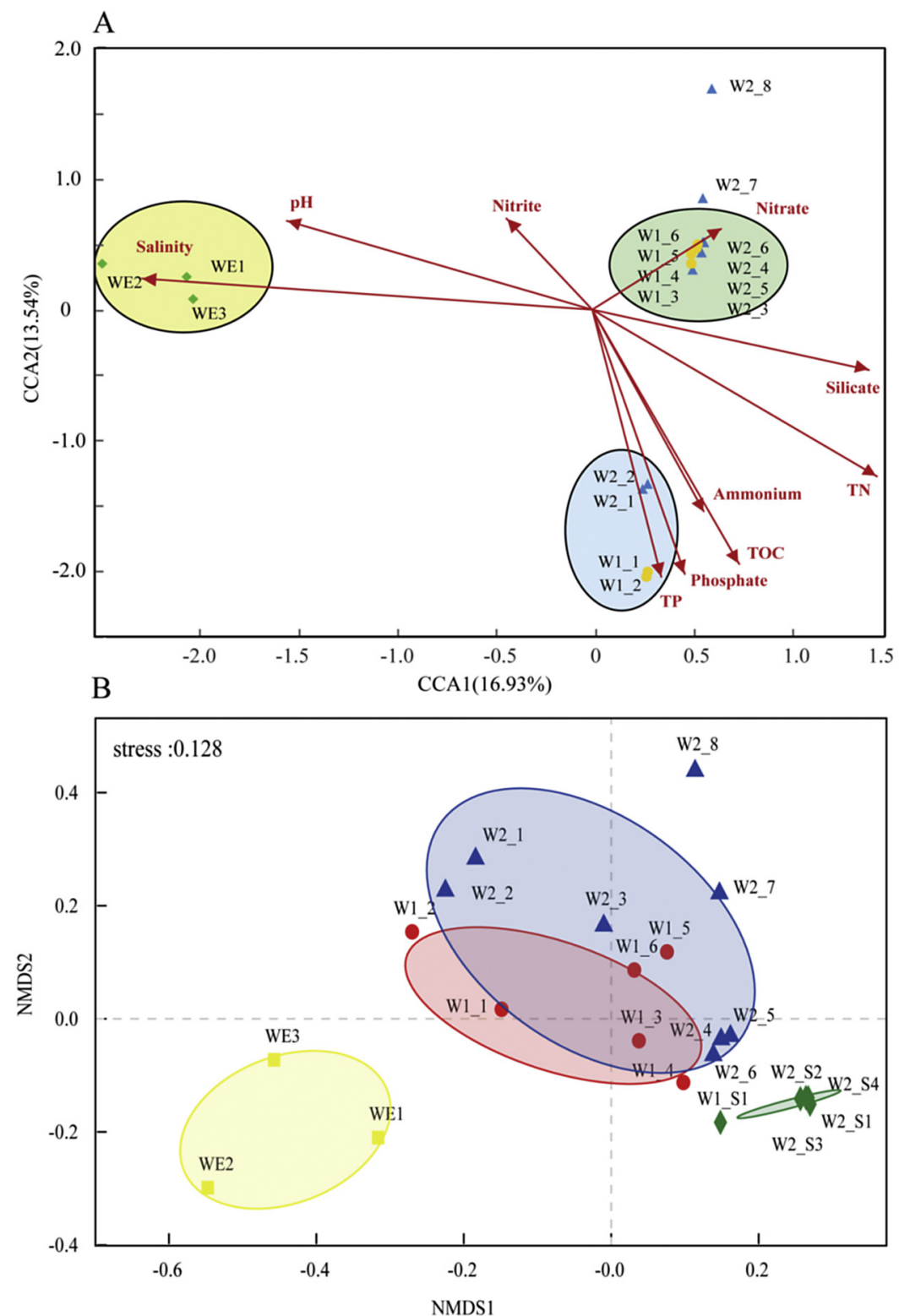

Fig. 6. Canonical correspondence analysis (CCA) analysis based on bacterial community, ARGs, intI1 and 16S rRNA gene (A). Non-metric multi-dimensional scaling (NMDS) analysis (Bray-Curtis distance) based on water quality parameters, bacterial community, ARGs, intI1 and 16S rRNA gene (B).

the CAS system. This phenomenon indicated that ARGs, intI1 and $16 \mathrm{~S}$ rRNA gene could be reduced efficiently in the IMR system for wastewater reclamation.

\subsection{Distribution of bacterial communities in the IMR system}

The diversity index showed a quite different pattern of bacterial community diversity across different samples (Wang et al., 2018). Community richness estimators (Sobs, Chao1 and ACE), community diversity estimators (Shannon and Simpson) and coverage were listed in Table S3. The coverage was in the range of $98.97 \%-99.4 \%$, which revealed that the high-throughput sequencing depth was enough to cover the most of communities as previous reports (Zhao et al., 2018a). In the IMR system, the community richness estimators decreased sharply after the treatment of microfiltration (W2-7) and RO filtration (W2-8). The community diversity estimators also illustrated that the diversity of water samples after the process of microfiltration (W2-7) and RO filtration (W2-8) were obviously lower than that of other water samples. In contrary, the $\alpha$-diversity estimators showed that there was no significant reduction of the richness and diversity in the CAS system.

Bacterial communities on phylum level (Fig. S1) and genus level (Fig. S2) were graphically demonstrated in bar charts. There were obvious differences in the bacterial communities of wastewater, sludge and seawater samples. The relative abundance of Proteobacteria was the highest on phylum level, followed by Bacteroidetes, Actinobacteria, Acidobacteria, Chloroflexi, Firmicutes, Planctomycetes, Cyanobacteria, Nitrospirae, Synergistetes and so on. In the IMR system, the relative abundances of Proteobacteria, Bacteroidetes, Actinobacteria and Firmicutes were relatively higher in W2-1 and W2-2. The relative abundances of Acidobacteria, Planctomycetes and Nitrospirae increased in the anoxic, aerobic and microfiltration tanks. Interestingly, the relative abundances of Actinobacteria and Cyanobacteria increased after the microfiltration and RO filtration processes, while the phyla of Acidobacteria, Chloroflexi, Firmicutes, Planctomycetes and Nitrospirae decreased obviously. In total, the relative abundances of Firmicutes and Synergistetes decreased, while the abundances of Actinobacteria and Cyanobacteria increased throughout the whole processes of the IMR system. It was worthy noted that Cyanobacteria was detected with high 


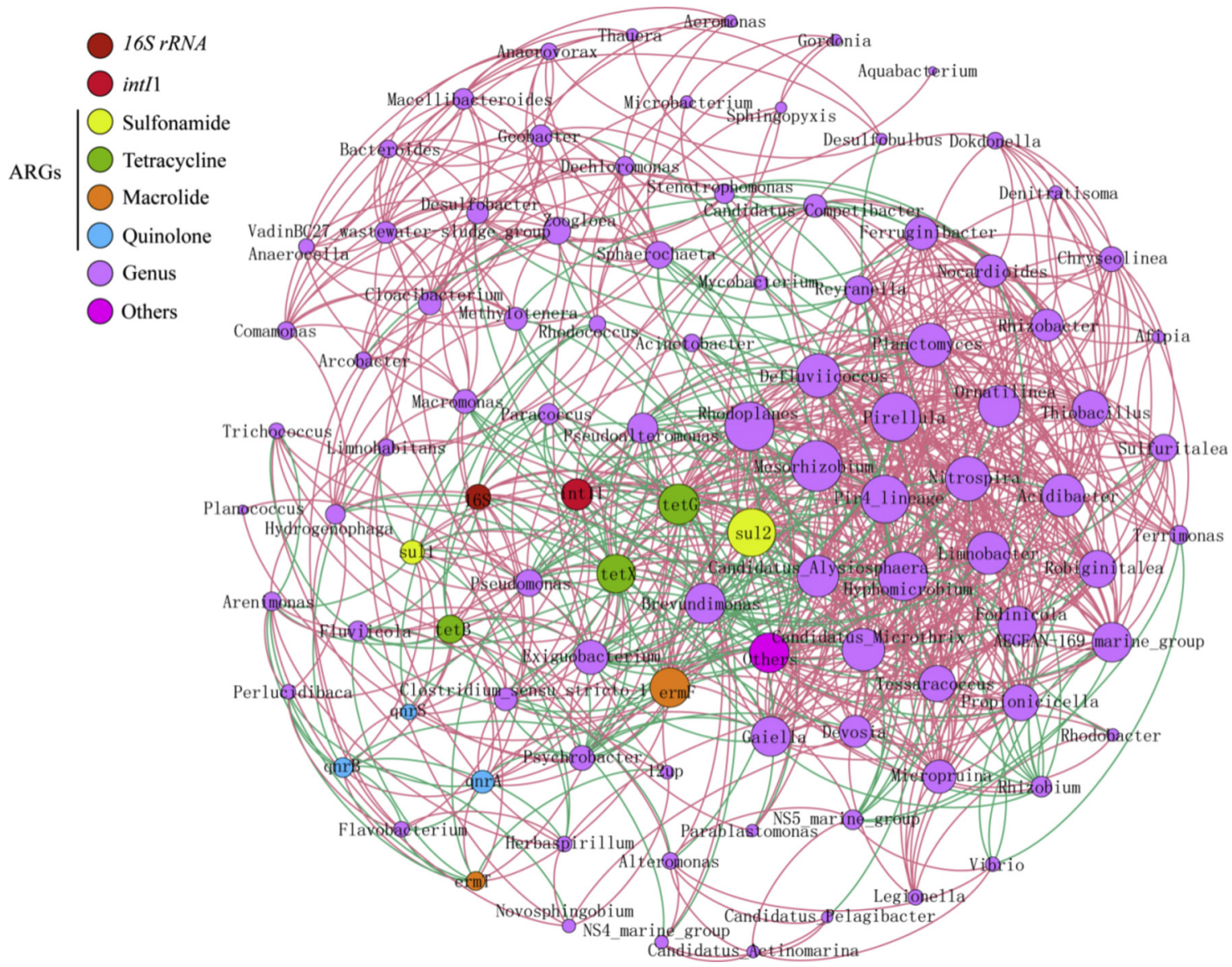

Fig. 7. Network analysis of ARGs, intI1, 16S rRNA and top 150 bacteria genera. Nodes were colored according to bacteria genus and types of genes. The strong (Spearman's correlation coefficient $>0.6)$ and significant $(\mathrm{p}<0.01)$ correlation was demonstrated by connections (red, positive correlation; green, negative correlation). The size of nodes was proportional to the number of connections. Others, unclassified and norank bacteria in top 150 bacteria genera (For interpretation of the references to colour in this figure legend, the reader is referred to the web version of this article).

relative abundances in receiving seawater samples, which was consistent with the bacterial communities of the effluent after microfiltration process.

The top 87 bacterial genera of all samples were shown in Fig. S2. In the surrounding sea of the WWTPs, Pseudoalteromonas accounted for the largest percentage, followed by genera of Fluviicola, Pelagibacter, Psychrobacter, Actinomarina, Flavobacteriaceae, Alteromonas, Synechococcus, Salinirepens, Aquabacterium and so on. In the raw influent of the IMR system, Comamonas, Macellibacteroides, Cloacibacterium, Rhodococcus, Pseudomonas, Bacteroides, Trichococcus, Geobacter, Desulfobacter and Fulvimarina were the predominant genera, while they disappeared gradually or were detected with low relative abundances after the treatment of microfiltration and RO filtration. This result indicated that the IMR system contributed to the decrease of the bacterial diversity, while the bacterial diversity after the CAS system did not decrease.

\subsection{Correlations of ARGs, bacterial communities and environmental factors}

CCA analysis (Luo et al., 2017) among water quality parameters (salinity, $\mathrm{pH}$, ammonium, nitrate, nitrite, phosphate, silicate, TN, TP and TOC), antibiotic resistant genes and bacterial communities were demonstrated in Fig. 6A. The result indicated that the bacterial communities of the seawater samples (WE1, WE2 and WE3) were positively affected by salinity and $\mathrm{pH}$, in accordance with previous research which demonstrated that high salinity may be used as a potential method for the removal of ARGs and bacteria carrying ARGs (Liu et al., 2018). The wastewater samples collected from the raw influent and primary clarify unit were positively influenced by TP, TOC, ammonium and phosphate. The result of CCA analysis demonstrated the strong clustering of different samples to separate reuse water and the effluent of microfiltration from other water samples.

NMDS analysis between all 22 samples was performed using braycurtis distances (Zhao et al., 2018b). As shown in Fig. 6B, a strong clustering of the detected ARGs, intI1, 16S rRNA gene and established microbial communities were constructed according to their abundances. The clustering significantly separated seawater samples and sludge samples from the wastewater samples in the WWTPs. Most of all, the reuse water (W2-8) and the effluent of microfiltration (W2-7) were significantly separated from other water samples in the WWTPs. The result of NMDS analysis was well corresponded with the clustering result of CCA analysis.

\subsection{Co-occurrence patterns among ARGs, intI1 and bacterial communities}

Co-occurrence patters of ARGs, intI1, 16S rRNA gene and bacterial communities were generally investigated by network analysis to reveal the bacterial genera carrying ARGs and intI1 (Guo et al., 2017). Connections of significant $(\mathrm{p}<0.01)$ and strong (Spearman's correlation coefficient $>0.6$ ) correlations were identified among the top 150 bacterial genera, ten ARGs, intI1 and 16S rRNA gene (Fig. 7).

ARGs of sul2, tet $\mathrm{G}$, tetX and erm $\mathrm{F}$ hold the most frequent correlations with other genes and bacterial genera. Genera of Rhodoplanes, Mesorhizobium, Pirellula and Pir4_lineage hold the most frequent correlations with other genes and genera. Interestingly, 16S rRNA gene was positively correlated with most of ARGs (sul, tet, ermF and qnrA), intI1 
and a few genera of bacteria (Defluviicoccus, Competibacter, Trichococcus and Clostridium, $\mathrm{p}<0.01$ ). The intI1 gene was positively correlated with all detected ARGs, except ermT and qnrB, which suggested that intI1 played important roles in the propagation of ARGs. Previous studies also presented that there were not positive and significant correlation between intI1 and ermT/qnrB (Chen et al., 2019; Duan et al., 2018; Hu et al., 2019). Meanwhile, intI1 was significantly related to the bacterial genera of Nitrospira, Defluviicoccus, Competibacter, Planctomyces, Alysiosphaera, Pirellula, Rhodoplanes and Clostridium. Additionally, there were strong (Spearman's correlation coefficient > $0.77)$ and significant $(\mathrm{p}<0.01)$ correlations between sulfonamides antibiotic resistance genes (sul1 and sul2) and tetracyclines antibiotic resistance genes (tet $\mathrm{B}$, tet $\mathrm{G}$ and tet $\mathrm{X}$ ).

Seven genes out of all the detected ARGs and intI 1 were assigned to Clostridium, while six genes were assigned to Defluviicoccus. Moreover, five genes were assigned to Pirellula, Pir4_lineage, Alysiosphaera, Competibacter, Rhodoplanes and Microthrix, while four genes were assigned to Trichococcus, Tessaracoccus, Ornatilinea, Hyphomicrobium, Mesorhizobium and Nitrospira with positive correlations. All of these genera might carry multiple antibiotic resistance genes and be resistant to multiple antibiotics. Furthermore, these bacteria genera, detected in the effluent of AAO treatment with relatively high abundances, were not detected in the water samples after microfiltration and RO filtration or detected with lower relative abundances. For example, Clostridium, which was the potential multi-antibiotic resistant bacteria, accounted for $0.35 \%$ in the raw influent and $0.01 \%$ in the effluent of the IMR system. Defluviicoccus was also the potential multi-antibiotic resistant bacteria. It was detected with the percentage of $1.26 \%$ in the water after AAO treatment, and only accounted for $0.01 \%$ in the reuse water. In summary, potential multi-antibiotic resistant bacteria could be reduced effectively by microfiltration and RO filtration processes in the IMR system, while the removal efficiencies of these genera were much lower in the CAS system.

\section{Conclusions}

Ten types of ARGs, intI1 and 16S rRNA gene were detected and quantified in the reclaimed water reuse system with integrated membrane process in a full-scale WWTPs. Results indicated that ARGs, intI1 and 16S rRNA gene could be reduced efficiently in the reclaimed water reuse system with integrated membrane process, while the removal efficiencies of these genes were lower in the CAS system. CCA and NMDS analysis demonstrated the strong clustering which separated the reuse water from other water samples in the WWTPs. Network analysis revealed the occurrence of potential multi-antibiotic resistant bacteria. In comparison with conventional wastewater treatment system, the potential multi-antibiotic resistant bacteria could be removed effectively by microfiltration and RO filtration processes in the IMR system. These findings demonstrate that the integrated membrane process is a significant and efficient approach for the removal of ARGs and multiantibiotic resistant bacteria.

\section{Declaration of Competing Interest}

The authors declare no competing interest.

\section{Acknowledgements}

This work was supported by National Natural Science Foundation of China (No. 41877131), Taishan Scholar Program of Shandong Province (No. tsqn201812116), One Hundred Talents Program of Chinese Academy of Sciences (No. Y629041021), and Two-Hundred Talents Plan of Yantai (No. Y739011021).

\section{Appendix A. Supplementary data}

Supplementary material related to this article can be found, in the online version, at doi:https://doi.org/10.1016/j.jhazmat.2019.121025.

\section{References}

Auerbach, E.A., Seyfried, E.E., McMahon, K.D., 2007. Tetracycline resistance genes in activated sludge wastewater treatment plants. Water Res. 41, 1143-1151.

Berendonk, T.U., Manaia, C.M., Merlin, C., Fatta-Kassinos, D., Cytryn, E., Walsh, F. Bürgmann, H., Sørum, H., Norström, M., Pons, M.-N., Kreuzinger, N., Huovinen, P., Stefani, S., Schwartz, T., Kisand, V., Baquero, F., Martinez, J.L., 2015. Tackling antibiotic resistance: the environmental framework. Nat. Rev. Microbiol. 13, 310.

Chen, H., Bai, X., Li, Y., Jing, L., Chen, R., Teng, Y., 2019. Characterization and sourcetracking of antibiotic resistomes in the sediments of a peri-urban river. Sci. Total Environ. 679, 88-96.

Chen, H., Zhang, M., 2013. Occurrence and removal of antibiotic resistance genes in municipal wastewater and rural domestic sewage treatment systems in eastern China. Environ. Int. 55, 9-14.

Coutinho de Paula, E., Amaral, M.C.S., 2017. Extending the life-cycle of reverse osmosis membranes: a review. Waste Manag. Res. 35, 456-470.

Duan, M., Gu, J., Wang, X., Li, Y., Zhang, S., Yin, Y., Zhang, R., 2018. Effects of genetically modified cotton stalks on antibiotic resistance genes, intI1, and intI2 during pig manure composting. Ecotoxicol. Environ. Saf. 147, 637-642.

Fiorentino, A., Esteban, B., Garrido-Cardenas, J.A., Kowalska, K., Rizzo, L., Aguera, A., Pérez, J.A.S., 2019. Effect of solar photo-Fenton process in raceway pond reactors at neutral $\mathrm{pH}$ on antibiotic resistance determinants in secondary treated urban wastewater. J. Hazard. Mater. 378, 120737.

Guo, J., Li, J., Chen, H., Bond, P.L., Yuan, Z., 2017. Metagenomic analysis reveals wastewater treatment plants as hotspots of antibiotic resistance genes and mobile genetic elements. Water Res. 123, 468-478.

Hu, Y., Jiang, L., Zhang, T., Jin, L., Han, Q., Zhang, D., Lin, K., Cui, C., 2018. Occurrence and removal of sulfonamide antibiotics and antibiotic resistance genes in conventional and advanced drinking water treatment processes. J. Hazard. Mater. 360, 364-372.

Hu, Q., Zhang, X.X., Jia, S., Huang, K., Tang, J., Shi, P., Ye, L., Ren, H., 2016. Metagenomic insights into ultraviolet disinfection effects on antibiotic resistome in biologically treated wastewater. Water Res. 101, 309-317.

Hu, Y., Zhang, T., Jiang, L., Luo, Y., Yao, S., Zhang, D., Lin, K., Cui, C., 2019. Occurrence and reduction of antibiotic resistance genes in conventional and advanced drinking water treatment processes. Sci. Total Environ. 669, 777-784.

Lan, L., Kong, X., Sun, H., Li, C., Liu, D., 2019. High removal efficiency of antibiotic resistance genes in swine wastewater via nanofiltration and reverse osmosis processes. J. Environ. Manage. 231, 439-445.

Le, T.H., Ng, C., Tran, N.H., Chen, H., Gin, K.Y.H., 2018. Removal of antibiotic residues, antibiotic resistant bacteria and antibiotic resistance genes in municipal wastewater by membrane bioreactor systems. Water Res. 145, 498-508.

Li, N., Sheng, G.P., Lu, Y.Z., Zeng, R.J., Yu, H.Q., 2017. Removal of antibiotic resistance genes from wastewater treatment plant effluent by coagulation. Water Res. 111, 204-212.

Li, B., Yang, Y., Ma, L., Ju, F., Guo, F., Tiedje, J.M., Zhang, T., 2015. Metagenomic and network analysis reveal wide distribution and co-occurrence of environmental antibiotic resistance genes. Int. Soc. Microb. Ecol. J. 9, 2490.

Liu, M., Li, Q., Sun, H., Jia, S., He, X., Li, M., Zhang, X.X., Ye, L., 2018. Impact of salinity on antibiotic resistance genes in wastewater treatment bioreactors. Chem. Eng. J. $338,557-563$

Lu, J., Zhang, Y., Wu, J., Wang, J., Zhang, C., Lin, Y., 2019. Occurrence and spatial distribution of antibiotic resistance genes in the Bohai Sea and Yellow Sea areas, China. Environ. Pollut. 252, 450-460.

Luo, Y., Mao, D., Rysz, M., Zhou, Q., Zhang, H., Xu, L., Alvarez, P.J.J., 2010. Trends in antibiotic resistance genes occurrence in the Haihe River, China. Environ. Sci. Technol. 44, 7220-7225.

Luo, G., Li, B., Li, L.G., Zhang, T., Angelidaki, I., 2017. Antibiotic resistance genes and correlations with microbial community and metal resistance genes in full-scale biogas reactors as revealed by metagenomic analysis. Environ. Sci. Technol. 51, 4069-4080.

Ma, Z., Wu, H., Zhang, K., Xu, X., Wang, C., Zhu, W., Wu, W., 2018. Long-term low dissolved oxygen accelerates the removal of antibiotics and antibiotic resistance genes in swine wastewater treatment. Chem. Eng. J. 334, 630-637.

Mao, D., Luo, Y., Mathieu, J., Wang, Q., Feng, L., Mu, Q., Feng, C., Alvarez, P.J.J., 2014 Persistence of extracellular DNA in river sediment facilitates antibiotic resistance gene propagation. Environ. Sci. Technol. 48, 71-78.

Marti, E., Variatza, E., Balcázar, J.L., 2014. Bacteriophages as a reservoir of extendedspectrum $\beta$-lactamase and fluoroquinolone resistance genes in the environment. Clin. Microbiol. Infect. 20, O456-459.

Michael, I., Rizzo, L., McArdell, C.S., Manaia, C.M., Merlin, C., Schwartz, T., Dagot, C., Fatta-Kassinos, D., 2013. Urban wastewater treatment plants as hotspots for the release of antibiotics in the environment: a review. Water Res. 47, 957-995.

Michael-Kordatou, I., Karaolia, P., Fatta-Kassinos, D., 2018. The role of operating parameters and oxidative damage mechanisms of advanced chemical oxidation processes in the combat against antibiotic-resistant bacteria and resistance genes present in urban wastewater. Water Res. 129, 208-230.

Proia, L., Anzil, A., Borrego, C., Farrè, M., Llorca, M., Sanchis, J., Bogaerts, P., Balcázar, J.L., Servais, P., 2018. Occurrence and persistence of carbapenemases genes in hospital and wastewater treatment plants and propagation in the receiving river. J. 
Hazard. Mater. 358, 33-43.

Pruden, A., Larsson, D.G.J., Amézquita, A., Collignon, P., Brandt Kristian, K., Graham David, W., Lazorchak James, M., Suzuki, S., Silley, P., Snape Jason, R., Topp, E., Zhang, T., Zhu, Y.G., 2013. Management options for reducing the release of antibiotics and antibiotic resistance genes to the environment. Environ. Health Perspect. 121, 878-885.

Su, J.Q., An, X.L., Li, B., Chen, Q.L., Gillings, M.R., Chen, H., Zhang, T., Zhu, Y.G., 2017. Metagenomics of urban sewage identifies an extensively shared antibiotic resistome in China. Microbiome 5, 84.

Tahrani, L., Soufi, L., Mehri, I., Najjari, A., Hassan, A., Van Loco, J., Reyns, T., Cherif, A., Mansour, H.B., 2015. Isolation and characterization of antibiotic-resistant bacteria from pharmaceutical industrial wastewaters. Microb. Pathog. 89, 54-61.

Wang, F.H., Qiao, M., Lv, Z.E., Guo, G.X., Jia, Y., Su, Y.H., Zhu, Y.G., 2014. Impact of reclaimed water irrigation on antibiotic resistance in public parks, Beijing, China. Environ. Pollut. 184, 247-253.

Wang, J.H., Lu, J., Zhang, Y.-X., Wu, J., Zhang, C., Yu, X., Zhang, Z., Liu, H., Wang, W.H., 2018. High-throughput sequencing analysis of the microbial community in coastal intensive mariculture systems. Aquac. Eng. 83, 93-102.

Xu, Y., Xu, J., Mao, D., Luo, Y., 2017. Effect of the selective pressure of sub-lethal level of heavy metals on the fate and distribution of ARGs in the catchment scale. Environ. Pollut. 220, 900-908.

Yi, Q., Zhang, Y., Gao, Y., Tian, Z., Yang, M., 2017. Anaerobic treatment of antibiotic production wastewater pretreated with enhanced hydrolysis: simultaneous reduction of COD and ARGs. Water Res. 110, 211-217.

Zhang, Q.Q., Ying, G.G., Pan, C.G., Liu, Y.S., Zhao, J.L., 2015. Comprehensive evaluation of antibiotics emission and fate in the river basins of China: source analysis, multimedia modeling, and linkage to bacterial resistance. Environ. Sci. Technol. 49, $6772-6782$.
Zhang, T., Hu, Y., Jiang, L., Yao, S., Lin, K., Zhou, Y., Cui, C., 2019. Removal of antibiotic resistance genes and control of horizontal transfer risk by UV, chlorination and UV/ chlorination treatments of drinking water. Chem. Eng. J. 358, 589-597.

Zhang, Y., Zhuang, Y., Geng, J., Ren, H., Xu, K., Ding, L., 2016. Reduction of antibiotic resistance genes in municipal wastewater effluent by advanced oxidation processes. Sci. Total Environ. 550, 184-191.

Zhang, W.J., Xu, X.R., Schwarz, S., Wang, X.M., Dai, L., Zheng, H.J., Liu, S., 2014. Characterization of the IncA/C plasmid pSCEC2 from Escherichia coli of swine origin that harbours the multiresistance gene cfr. J. Antimicrob. Chemother. 69, 385-389.

Zhao, R., Feng, J., Yin, X., Liu, J., Fu, W., Berendonk, T.U., Zhang, T., Li, X., Li, B., 2018a. Antibiotic resistome in landfill leachate from different cities of China deciphered by metagenomic analysis. Water Res. 134, 126-139.

Zhao, R., Feng, J., Yin, X., Liu, J., Fu, W., Berendonk, T.U., Zhang, T., Li, X., Li, B., 2018b. Antibiotic resistome in landfill leachate from different cities of China deciphered by metagenomic analysis. Water Res. 134, 126-139.

Zheng, J., Su, C., Zhou, J., Xu, L., Qian, Y., Chen, H., 2017. Effects and mechanisms of ultraviolet, chlorination, and ozone disinfection on antibiotic resistance genes in secondary effluents of municipal wastewater treatment plants. Chem. Eng. J. 317, 309-316.

Zhou, L.J., Ying, G.G., Zhao, J.L., Yang, J.F., Wang, L., Yang, B., Liu, S., 2011. Trends in the occurrence of human and veterinary antibiotics in the sediments of the Yellow River, Hai River and Liao River in northern China. Environ. Pollut. 159, 1877-1885.

Zhu, Y.G., Zhao, Y., Li, B., Huang, C.L., Zhang, S.Y., Yu, S., Chen, Y.S., Zhang, T., Gillings, M.R., Su, J.Q., 2017. Continental-scale pollution of estuaries with antibiotic resistance genes. Nat. Microbiol. 2, 16270.

Zhu, Y.G., Johnson, T.A., Su, J.Q., Qiao, M., Guo, G.X., Stedtfeld, R.D., Hashsham, S.A., Tiedje, J.M., 2013. Diverse and abundant antibiotic resistance genes in Chinese swine farms. Proc. Natl. Acad. Sci. U. S. A. 110, 3435-3440. 